\title{
AUMENTO DA TRANSPARÊNCIA PULMONAR NA INFÂNCIA: ENSAIO ICONOGRÁFICO*
}

\author{
Daniel Lahan Martins ${ }^{1}$, Inês Minniti Rodrigues Pereira ${ }^{2}$, Beatriz Regina Alvares², Lívio Nanni², \\ Jamal Baracat ${ }^{2}$
}

\begin{abstract}
Resumo Assimetrias torácicas representadas pelo aumento da transparência pulmonar são causas importantes de morbidade na população pediátrica. A avaliação dos pacientes afetados freqüentemente requer múltiplas modalidades de imagem para diagnosticar a anomalia e planejar a correção cirúrgica. Os autores analisam e ilustram os aspectos de assimetrias torácicas comuns e raras, com ênfase nas manifestações radiológicas. A aplicabilidade das diversas modalidades de imagem no diagnóstico e tratamento é avaliada. Diagnósticos diferenciais, assim como armadilhas e dificuldades diagnósticas, são incluídas.

Unitermos: Doenças torácicas; Malformação adenomatóide cística; Enfisema lobar congênito; Hérnia diafragmática congênita.
\end{abstract}

\begin{abstract}
Increased radiolucency of the lungs in infancy: iconographic essay.
Thoracic asymmetries characterized by increased radiolucency of the lungs are important causes of morbidity in infants and children. Evaluation of affected patients frequently requires multiple imaging methods to diagnose the abnormality and to plan the surgical correction. The authors review and illustrate the characteristics of both common and rare thoracic asymmetries with emphasis on the radiological findings. The usefulness of different imaging methods in the diagnosis and treatment of these conditions is evaluated. The differential diagnosis as well the pitfalls and diagnostic difficulties are also discussed.

Key words: Thoracic diseases; Congenital cystic adenomatoid malformation; Congenital lobar emphysema; Congenital diaphragmatic hernia.
\end{abstract}

\section{INTRODUÇÃO}

Diversas doenças se manifestam como lesões radiotransparentes assimétricas no tórax infantil, algumas delas letais. O reconhecimento da variada manifestação radiográfica facilita o diagnóstico imediato.

Malformação adenomatóide cística congênita (MACC), hérnia diafragmática congênita (HDC) e enfisema lobar congênito (ELC) podem ser condições de ameaça à vida que requerem correção cirúrgica, algumas vezes em situação emergencial. Enfisema pulmonar intersticial e pneumatoceles também podem ser letais, mas geralmente respondem a tratamento clínico. Hemitórax radiotransparente também é incluído nesta categoria e pode ser decorrente de pneumotórax, oclusão brônquica

\footnotetext{
* Trabalho realizado no Departamento de Radiologia da Faculdade de Ciências Médicas da Universidade Estadual de Campinas (Unicamp), Campinas, SP.

1. Médico Residente do Departamento de Radiologia da Unicamp.

2. Professores Doutores do Departamento de Radiologia da Unicamp.

Endereço para correspondência: Dr. Daniel Lahan Martins. Rua Manoel José Vilaça, 862. Bragança Paulista, SP, 12902-160. E-mail: daniel_lahan@hotmail.com

Recebido para publicação em $7 / 3 / 2005$. Aceito, após revisão, em 18/5/2005.
}

parcial ou hiperinsuflação compensatória de pulmão (sem obstrução) $)^{(\mathbf{1 , 2})}$.

Condições que se manifestam como dispnéia em recém-nascidos podem necessitar cirurgia ou procedimento intervencionista. A radiografia de tórax geralmente permite um diagnóstico precoce e acurado. Casos que necessitam de tratamento clínico geralmente têm acometimento bilateral. Casos cirúrgicos geralmente têm acometimento unilateral e efeito de massa ${ }^{(3,4)}$.

Existem condições encontradas incidentalmente nos estudos por imagem que não requerem intervenção imediata.

Apresentamos diversos achados radiográficos destas condições e sugerimos um algoritmo diagnóstico, apontando os diferentes diagnósticos diferenciais com casos ilustrados.

\section{MALFORMAÇÃO ADENOMATÓIDE CÍSTICA CONGENITA}

MACC dos pulmões é causa incomum de anomalia do desenvovimento em que há um aprisionamento da maturação pulmonar, que se pensa ser secundária a atresia brônquica primária ou falha na segmenta- ção brônquica normal e subsequente desenvolvimento de tecido pulmonar displásico distal ao segmento afetado ${ }^{(\mathbf{5 , 6})}$.

Nem todos pacientes com MACC são sintomáticos ao nascimento, alguns têm apresentação tardia e diagnóstico somente na vida adulta. O ultra-som pré-natal (US) é um meio de o radiologista identificar MACC na população de crianças assintomáticas na infância ${ }^{(7)}$.

A alteração é geralmente unilateral, envolvendo um ou mais lobos, mas acometimento bilateral tem sido descrito. A manifestação é variável (Figuras 1 e 2), mas os três tipos principais têm sido descritos: 1) uma única e extensa área predominantemente cística; 2) múltiplas áreas císticas de tamanhos variados $(0,5-1,5 \mathrm{~cm}) ; 3)$ lesão sólida ${ }^{(\mathbf{1 , 4}, \mathbf{8})}$. Quando existe preenchimento completo das áreas císticas, a radiografia demonstra um hemitórax opacificado, o que ocorre no momento do nascimento ${ }^{(9)}$. Embora seja importante reconhecer esta classificação morfológica para a compreensão do espectro clínico e patológico desta doença, o reconhecimento do tipo patológico de MACC não é clinicamente importante porque o resultado, na 


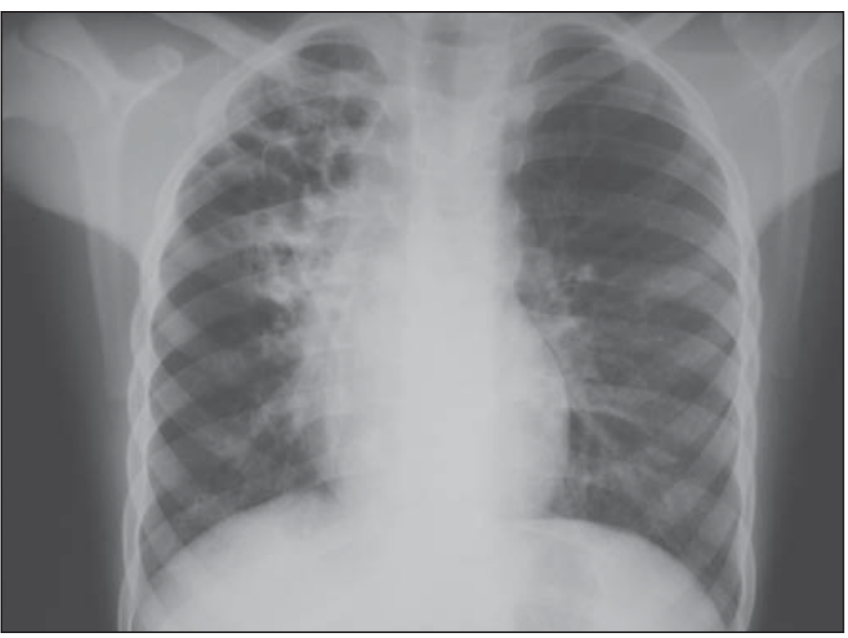

A

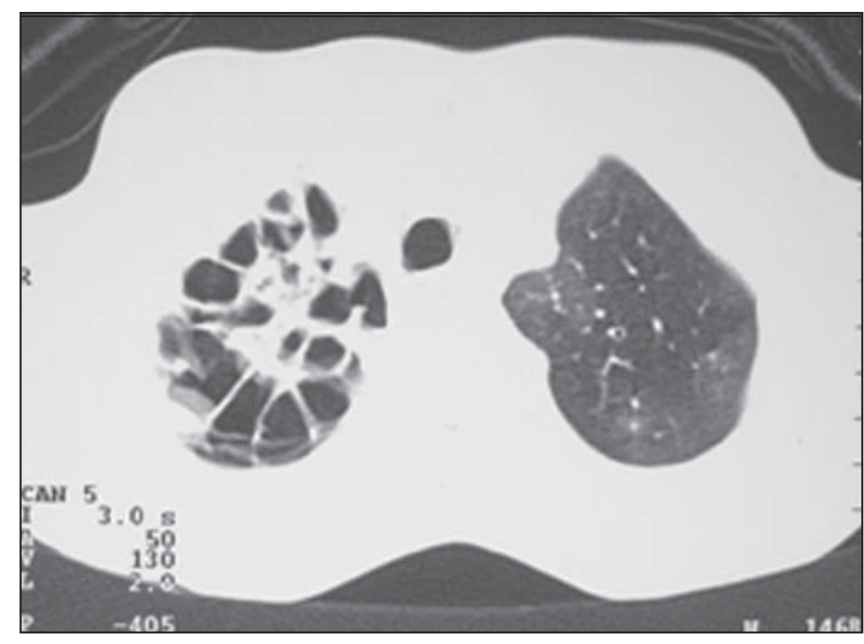

C

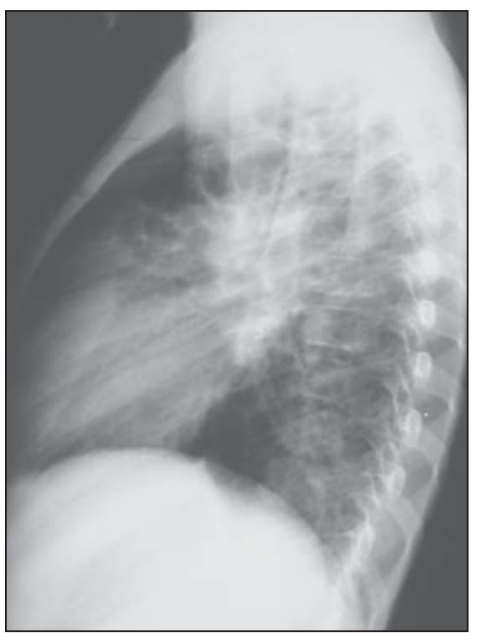

B

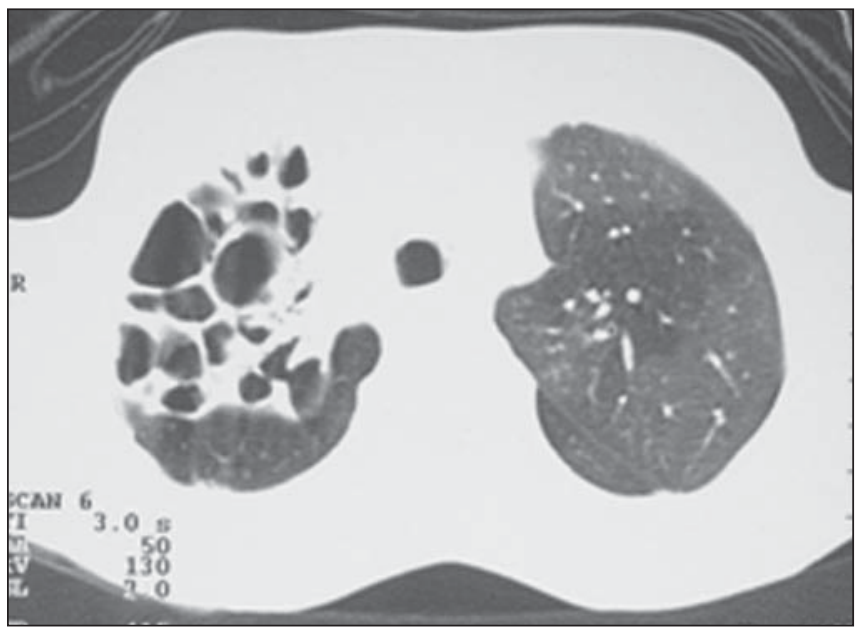

D

Figura 1. Paciente de nove anos de idade com MACC. Radiografias $(\mathbf{A}, \mathbf{B})$ e tomografias computadorizadas $(\mathbf{C}, \mathbf{D})$ de tórax mostram múltiplas áreas císticas no lobo superior direito.

maioria dos casos, é a ressecção cirúrgica. Ademais, existem pacientes com MACC que se apresentam com lesões histopatologicamente mistas dos tipos 1 e $2^{(5)}$.

A tomografia computadorizada (TC) é exame valioso antes da cirurgia, no sentido de acessar o grau de envolvimento do pulmão ipsilateral e para diagnosticar áreas de possível envolvimento do pulmão contralateral $^{(4)}$.

\section{HÉRNIA DIAFRAGMÁTICA CONGÊNITA}

O hemitórax assimétrico causado por HDC usualmente é visto em recém-nascidos com defeito póstero-lateral esquerdo do diafragma, conhecido como hérnia de Bochdalek $^{(4)}$.
Estes defeitos ocorrem no lado esquerdo em $85 \%$ a $90 \%$ dos $\operatorname{casos}^{(\mathbf{1 0 )}}$. Um efeito de massa da alça intestinal herniada pode prejudicar a ramificação e o desenvolvimento normal das vias aéreas e vasos, tanto ipsilateralmente como no pulmão contralateral.

Com o aumento no diagnóstico prénatal pelo US, terapias clínicas e cirúrgicas têm sido planejadas e realizadas com sucesso $^{(\mathbf{1 1})}$. O diagnóstico radiológico pósnatal geralmente não é difícil, e seus achados ajudam a predizer a sobrevivência em neonatos com $\mathrm{HDC}^{(\mathbf{1 2})}$.

As chaves no diagnóstico radiográfico incluem identificação de alças intestinais no tórax e abdome escavado secundário a herniação do seu conteúdo para o tórax (Figuras 3A,B e 4A,B). Daí a importância de se incluir o abdome no filme. Exame contrastado do trato gastrintestinal geralmente não é necessário para o diagnóstico (Figura 4C,D) ${ }^{(4)}$.

\section{ENFISEMA LOBAR CONGÊNITO}

ELC é caracterizado por distensão progressiva de um, às vezes dois lobos pulmonares. Acredita-se resultar de mecanismo valvulado brônquico, que causa hiperinsuflação progressiva do pulmão por permitir entrada de ar na inspiração ${ }^{(\mathbf{1 3})}$.

Em geral é diagnosticado no período neonatal ou na infância e é mais comum no sexo masculino do que no sexo feminino (3:1). O lobo superior esquerdo e o lobo médio são localizações usuais. Lobos inferiores raramente são afetados ${ }^{(14)}$. Os 
Figura 2. Radiografias de tórax em pósteroanterior (A) e perfil (B) de criança com MACC evidenciam múltiplas áreas císticas causando desvio mediastinal contralateral.

Figura 3. Hérnia diafragmática de Morgagni. Radiografias de tórax em ântero-posterior (A) e perfil (B) de uma criança com hemitórax direito cístico e desvio contralateral das estruturas mediastinais.

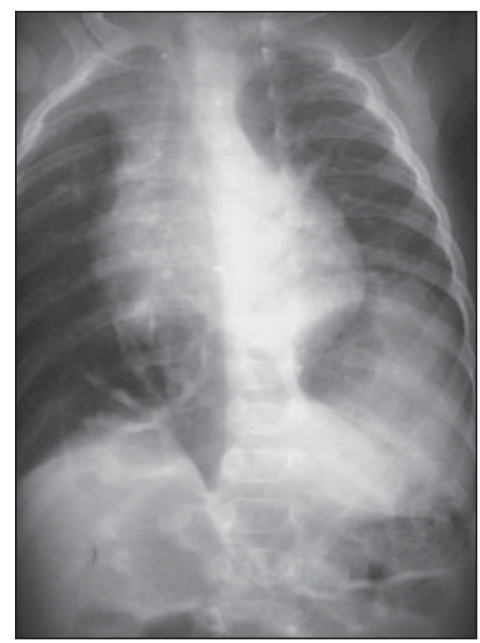

A

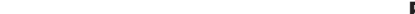

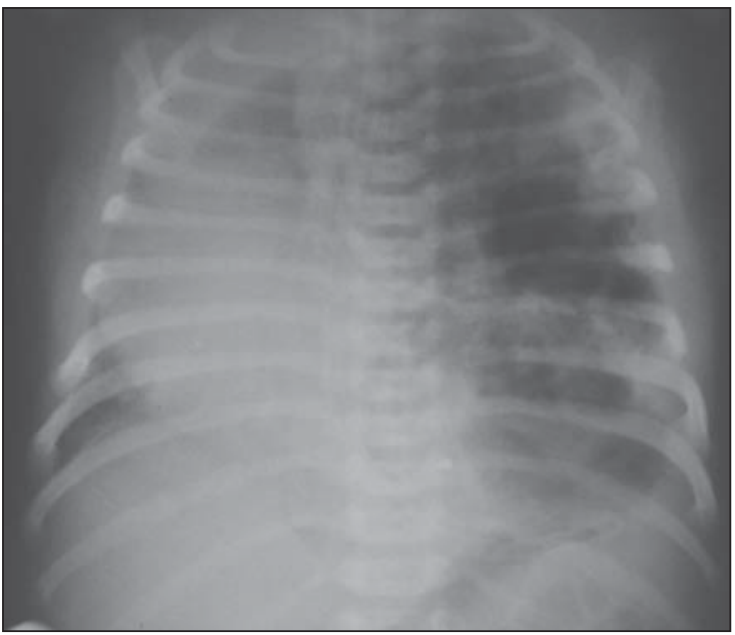

A

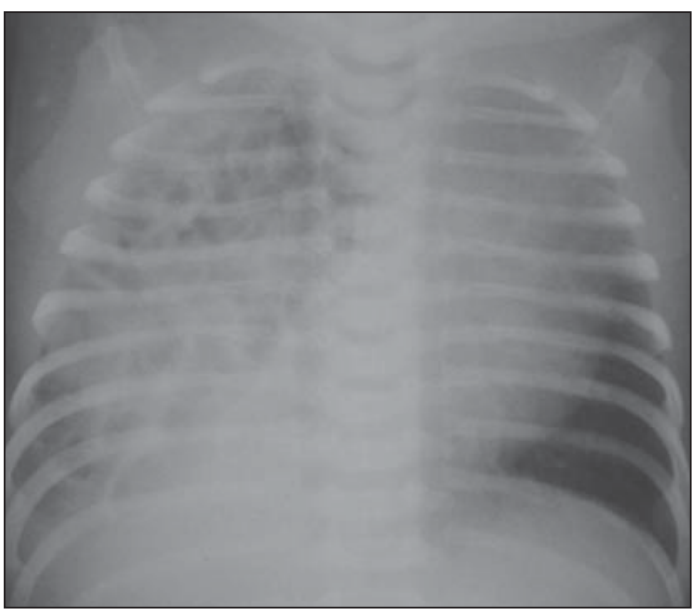

A
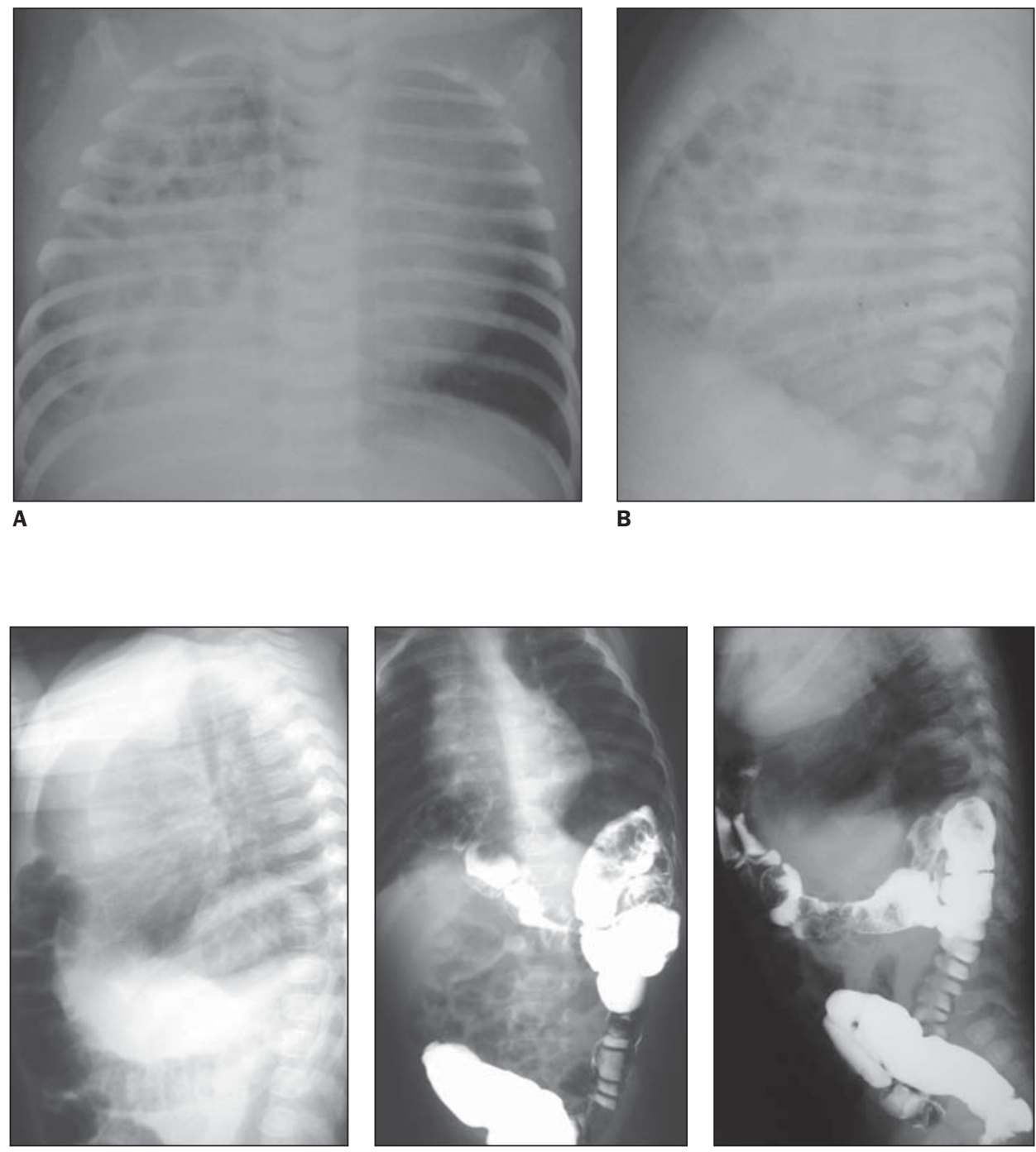

C

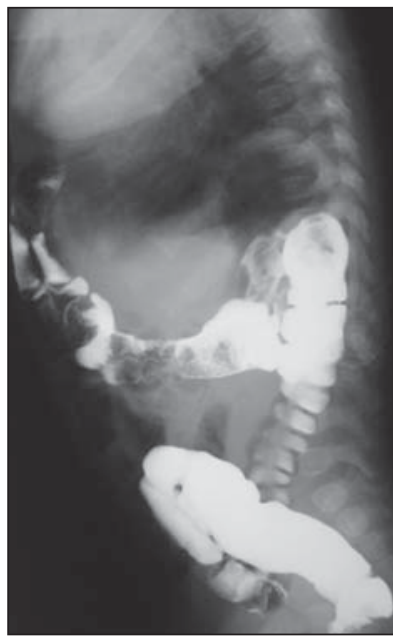

D

Figura 4. Hérnia diafragmática anterior. Radiografias de tórax em ântero-posterior (A) e perfil (B) mostram hemitórax direito com lesão cística volumosa. A radiografia em perfil evidencia abdome escavado, indicando hérnia. Enema com contraste (C,D) demonstra o cólon herniado através do diafragma. 
achados radiológicos são hiperinsuflação com retenção de ar na expiração (Figura 5).

\section{ENFISEMA PULMONAR INTERSTICIAL}

Bebês com ventilação com pressão positiva podem desenvolver enfisema intersticial de aparência multicística. Os cistos têm paredes finas e aparência de múltiplas pneumatoceles (Figura 6), ocasionalmente produzindo efeito de massa ${ }^{(4)}$.

\section{PNEUMATOCELES}

Pneumatoceles são coleções de ar com paredes finas que se desenvolvem como lesões pós-inflamatórias (Figura 7), freqüen- temente após pneumonia estafilocócica ${ }^{(\mathbf{1})}$. Também podem ser vistas após trauma e geralmente resolvem espontaneamente. Podem romper e produzir pneumotórax.

\section{PNEUMOTÓRAX}

Quando os contornos do pulmão são vistos delineados por ar intrapleural, é feito o diagnóstico de pneumotórax. Um hemitórax pode aparecer maior e mais radiotransparente que o outro com áreas de aparência cística. Pneumotórax hipertensivo é suspeitado quando existe efeito de massa causando desvio mediastinal, compressão pulmonar e distorção diafragmática (Figura 8). Decúbito lateral com raios horizontais permite diagnóstico correto.

\section{HIPERINSUFLAÇÃO COMPENSATÓRIA}

Hiperinsuflação compensatória de pulmão normal com desvio mediastinal em hemitórax opacificado pode ser visto em crianças com aspiração por corpo estranho (Figura 9), agenesia pulmonar, hipoplasia pulmonar, síndrome da cimitarra e sequiestro pulmonar, produzindo um tórax de aparência cística $^{(4,15)}$.

\section{CONCLUSÃO}

Os aspectos radiológicos de tórax assimétrico pediátrico são marcados por uma ênfase em diagnóstico emergencial, pois muitas das doenças que assim se manifes-

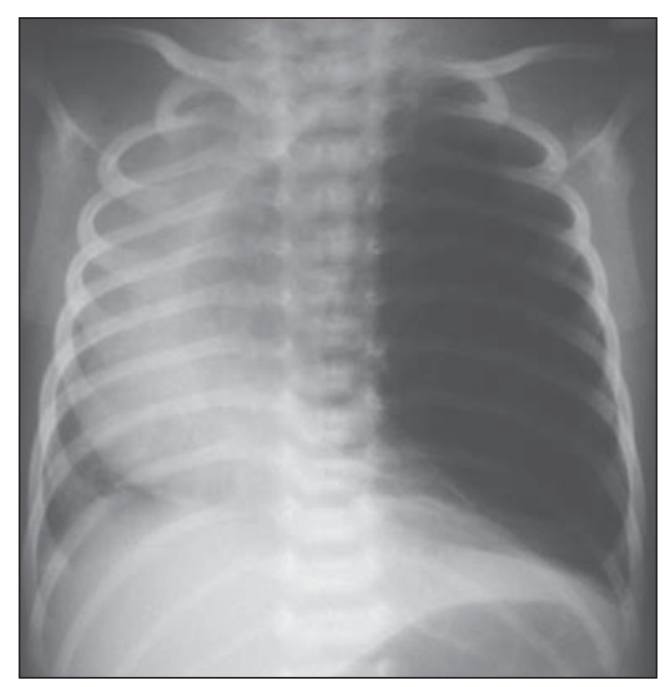

5

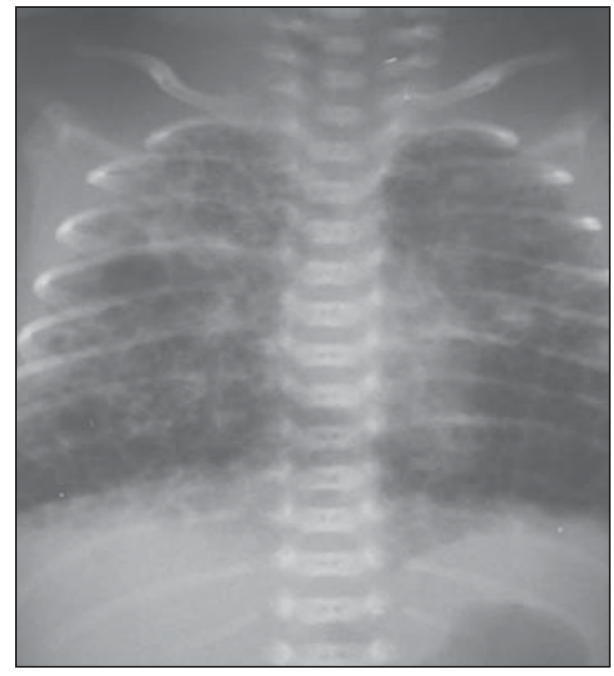

6

Figura 5. Enfisema lobar congênito. Radiografia de tórax em ântero-poste rior evidencia volumoso hemitórax esquerdo radiotransparente em neonato.

Figura 6. Enfisema pulmonar intersticial. Lesões císticas torácicas bilaterais em neonato submetido a ventilação por pressão positiva.

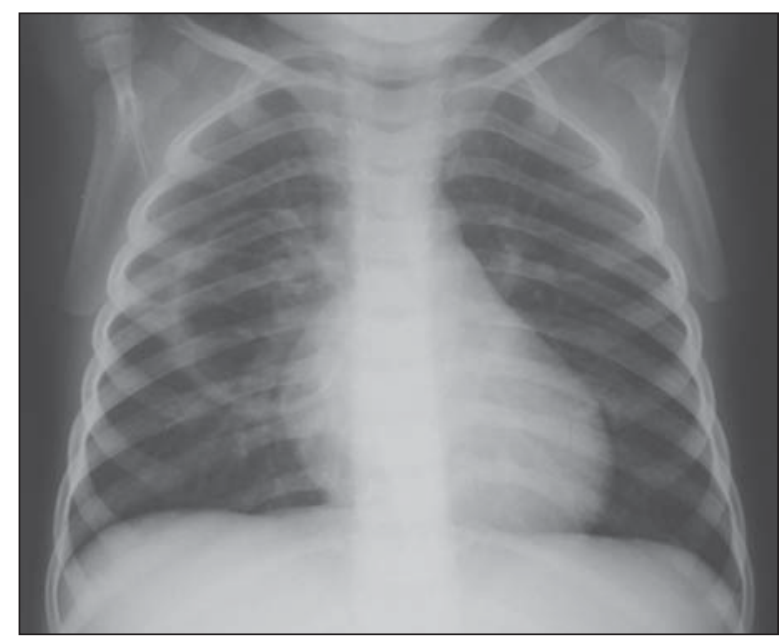

A

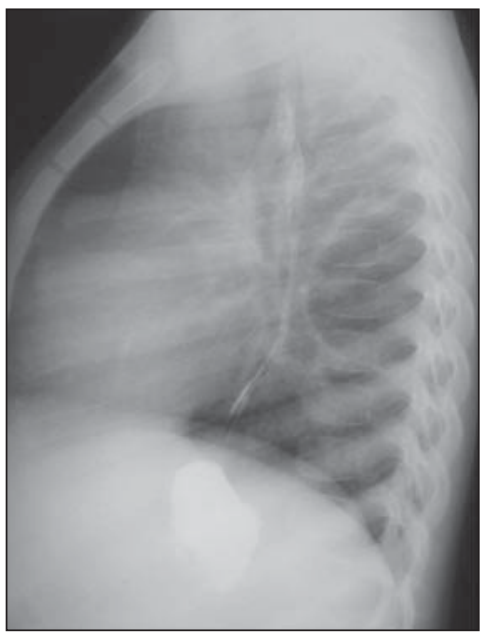

B
Figura 7. Pneumatocele. Radiografias de tórax em ântero-posterior (A) e perfil (B) mostram lesão cística única no hemitórax direito de criança após tratamento por pneumonia estafilocócica. 


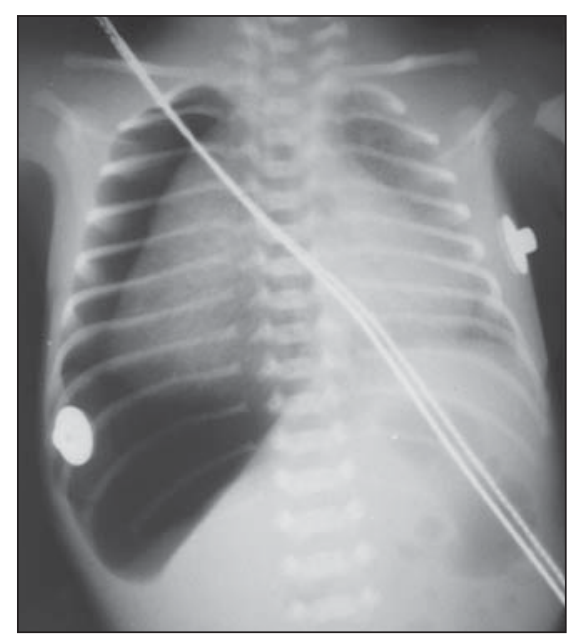

Figura 8. Pneumotórax hipertensivo. Radiografia de tórax em ântero-posterior evidencia grande quantidade de ar coletado no espaço pleural direito, com efeito de massa e desvio mediastinal contralateral.

tam são ameaçadoras à vida. Interpretação acurada da radiografia de tórax é essencial para o diagnóstico precoce, disponibilizando informações necessárias no amplo espectro de diagnósticos diferenciais.

\section{REFERÊNCIAS}

1. Alford BA, McIlhenny J, Jones JE, et al. Asymmetric radiographic findings in the pediatric chest: approach to early diagnosis. RadioGraphics 1993;13 77-93.

2. Tander B, Yalcin M, Yilmaz B, Ali Karadag C, Bulut M. Congenital lobar emphysema: a clinicopathologic evaluation of 14 cases. Eur J Pediatr Surg 2003;13:108-111.

3. Panicek DM, Heitzman ER, Randall PA, et al. The continuum of pulmonary developmental anomalies. RadioGraphics 1987;7:747-772.

4. Alford BA, McIlhenny J. An approach to the asymmetric neonatal chest radiograph. Radiol Clin North Am 1999;37:1079-1092.

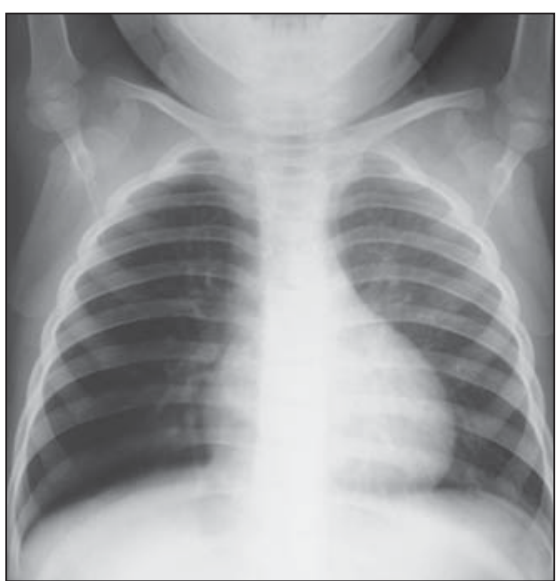

A

Figura 9. Radiografias de tórax em ântero-posterior na inspiração (A) e expiração (B) mostram hemitórax direito radiotransparente por mecanismo valvulado em criança com aspiração de corpo estranho.

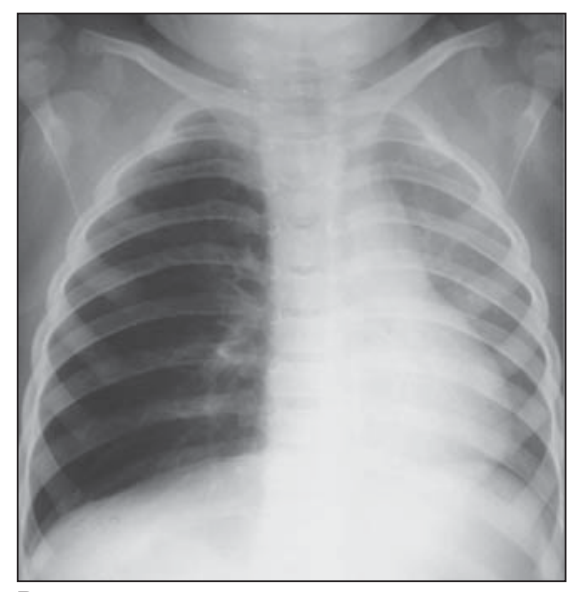

B
5. Kim WS, Lee KS, Kim IO, et al. Congenital cystic adenomatoid malformation of the lung: CT-pathologic correlation. AJR 1997;168:47-53.

6. Moerman P, Fryns JP, Vandenberghe K, Devlieger H, Lauweryns JM. Pathogenesis of congenital cystic adenomatoid malformation of the lung. Histopathology 1992;21:315-321.

7. Marshall KW, Blane CE, Teitelbaum DH, van Leeuwen K. Congenital cystic adenomatoid malformation: impact of prenatal diagnosis and changing strategies in the treatment of the asymptomatic patient. AJR 2000;175:1551-1554

8. Heij HA, Ekkelkamp S, Vos A. Diagnosis of congenital cystic adenomatoid malformation of the lung in newborn infants and children. Thorax 1990;45:122-125.

9. Rosado-de-Christenson ML, Stocker JT. Congenital cystic adenomatoid malformation. RadioGraphics 1991;11:865-886.

10. Harrison MR, de Lorimier AA. Congenital diaphragmatic hernia. Surg Clin North Am 1981;61: $1023-1035$
11. Karamanoukian HL, O'Toole SJ, Glick PL. "Stateof-the-art" management strategies for the fetus and neonate with congenital diaphragmatic hernia. J Perinatol 1996;16:S40-47.

12. Donnelly LF, Sakurai M, Klosterman LA, Delong DM, Strife JL. Correlation between findings on chest radiography and survival in neonates with congenital diaphragmatic hernia. AJR 1999;173: 1589-1593.

13. Karnak I, Senocak ME, Ciftci AO, Buyukpamukcu N. Congenital lobar emphysema: diagnostic and therapeutic considerations. J Pediatr Surg 1999;34: 1347-1351.

14. Zylak CJ, Eyler WR, Spizarny DL, Stone CH. Developmental lung anomalies in the adult: radiologic-pathologic correlation. RadioGraphics 2002; 22 Spec No:S25-43.

15. Berrocal T, Madrid C, Novo S, Gutiérrez J, Arjonilla A, Gómez-León N. Congenital anomalies of the tracheobronchial tree, lung, and mediastinum: embryology, radiology, and pathology. RadioGraphics 2004;24:e17. 